\title{
MODELING OF ACOUSTIC PROCESSES IN SOLIDS BASED ON PARTICLE INTERACTION
}

\author{
Angela Kuzovova, ${ }^{1}$, Timur Muksunov \\ Tomsk State University, 634050, Tomsk, Russia
}

\begin{abstract}
An algorithm for the numerical propagation of acoustic waves in solids has been developed. The algorithm is based on the particles interaction model of in a tetrahedral crystal lattice. Numerical integration of Newton's equations in the calculation of particle trajectories is applied. The possibility of numerical modeling of wave diffraction processes on heterogeneities is shown. The resonant phenomena are observed in simulation of acoustic oscillations for an ultrasonic waveguide by means of proposed algorithm.
\end{abstract}

\section{Introduction}

Acoustic methods are widely used in physical research, in particular, they allow studying various phenomena in solids. The high sensitivity of ultrasonic waves to the presence of mechanical heterogeneities of the medium contributed to the development of ultrasonic flaw detection. Ultrasonic methods allow determining the presence and type of defects in materials that are "opaque" for optical and radio wave methods. Hence, the development of methods for numerical modeling of acoustic processes that take into account all possible wave effects and nonlinear processes is relevant. In the linear approximation, acoustic processes in a homogeneous medium are described by the wave equation. The solution of this equation makes it possible to calculate the field distribution in the medium by specified sources. To account for heterogeneities in the density and speed of sound in a medium, the wave equation is solved in the approximation of small perturbations or in the singlescattering approximation. Far from all effects can be taken into account in the linear approximation for a homogeneous background medium. To take into account nonlinear effects, the propagation of waves in heterogeneous media is necessary the application of numerical methods. Widely applied the method of finite differences in the time domain [1]. This method is based on the iterative calculation of the subsequent state on the basis of the previous, in the process of numerical integration of the wave differential equation. The finite element method is applies for the analysis of stationary fields in the frequency domain and requires the solution of large systems of linear algebraic equations and is applicable in the linear approximation, but allows to take into account the spatial inhomogeneity of the medium. The most common approach is the simulation of interacting particles of matter on the basis of Newton's equations [2, 3]. Together with this, similar approaches are used to model acoustic processes [4].

\footnotetext{
${ }^{1}$ Corresponding author: Ang_kuz93@mail
} 


\section{Particle model of solid media}

In this paper, we propose a method for numerical simulation of the propagation of acoustic waves in solids based on an array of particles in a tetrahedral equidistant grid and Newton's equation of motion. Through the partitioning of the entire set of particles into groups and the parallel calculation of the equations of motion by means of OpenCL, is provided the accelerated modeling of acoustic processes in a solid. The properties of matter are determined by the dependence of the force interaction of the particles on the distance between them and the mass of the particles. For the modeling of a solid body, the spatial arrangement of the particles presented in Figure 1 is considered, where the distance between all nearest particles is the same and equal to $d$. We assume that the particles interact only with the nearest neighbors. For the lattice under consideration, each particle will have 12 neighbors.

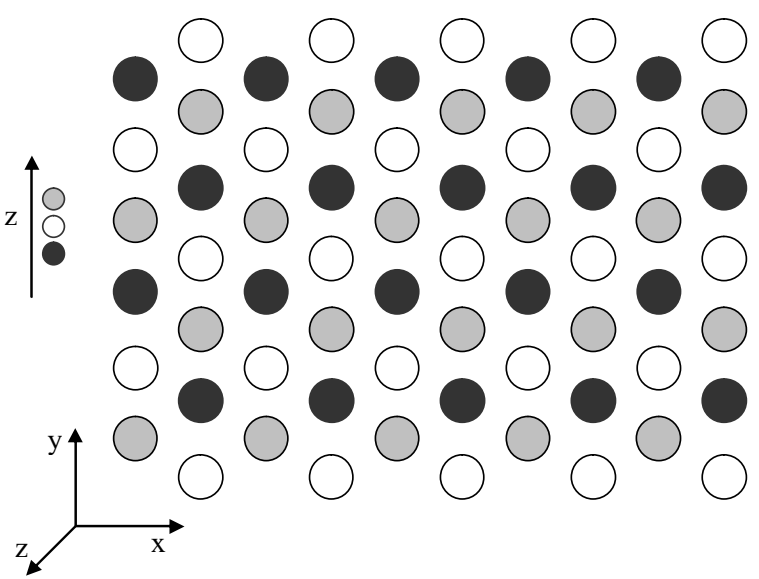

Fig. 1. Structure of the crystal lattice under consideration.

The interaction strength between the particles is given by the graph shown in Figure 2 and is determined by the formula (1):

$$
F(x)=(1-d / x)(d \sqrt{2}-x)^{2}(x<d \sqrt{2})
$$

where is $d$-the distance between the particles, $F(x)$ - the force of interaction between particles, $x$-particle coordinate (in Figure $2 \mathrm{x}$ is measured in values of $d$ ).

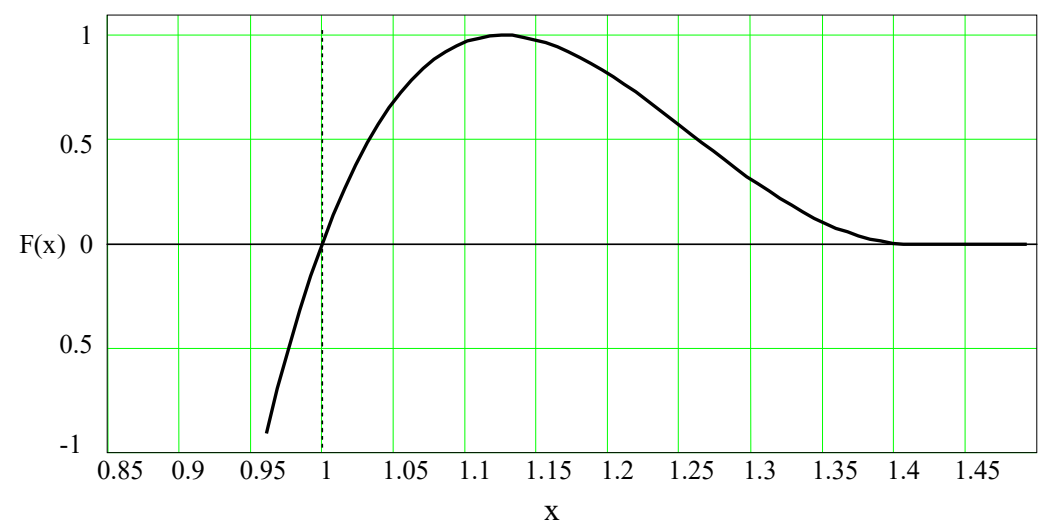

Fig. 2. Dependence of the attractive force between particles from distance. 
It is seen that for a distance $d$ (when $x=1$ ) between the particles, the interaction force is zero, and so the unperturbed structure will be stationary. Near the equilibrium point, the attractive force depends linearly on the distance, which allows modeling of linear acoustic processes and in the linear approximation to estimate the propagation velocity of longitudinal waves.

We consider a one-dimensional periodic chain of particles. Let the period of this chain be $d$. Then, in the state of equilibrium, the coordinate of the $n$-th particle of the chain is $x=n d$. When the particle is shifted from the equilibrium state, in the linear approximation, a force proportional to the magnitude of the shift appears (Hooke's law). We denote the shift of the $\mathrm{n}$-th particle from the equilibrium state by $X_{n}$. Hence the force acting on the particle on the left side can be written as $F_{1}=k\left(X_{n-1}-X_{n}\right)$, the force acting on the side of the right particle will be written as $F_{2}=k\left(X_{n+1}-X_{n}\right)$. For a central particle of mass $\mathrm{m}$, we can write Newton's equation in the form:

$$
m \frac{\partial^{2} X_{n}}{\partial t^{2}}=F_{1}+F_{2}=k\left(X_{n+1}-2 X_{n}+X_{n-1}\right)
$$

Let us consider the case when the step $d$ is much smaller than the characteristic distance, on which there is a significant change in the deviation of the particles (or much less than the wavelength). Then the quantity $\left(X_{n+1}-2 X_{n}+X_{n-1}\right) \approx d^{2} \frac{\partial^{2} X}{\partial x^{2}}$, therefore

$$
m \frac{\partial^{2} X_{n}}{\partial t^{2}}=k d^{2} \frac{\partial^{2} X}{\partial x^{2}}, \text { or } \frac{\partial^{2} X}{\partial x^{2}}-\frac{m}{k d^{2}} \frac{\partial^{2} X_{n}}{\partial t^{2}}=0
$$

- this is the canonical form of the wave equation, from which it follows that the speed of sound is determined by the expression: $\frac{1}{c^{2}}=\frac{m}{k d^{2}}$, therefore, the velocity of sound $c=d \sqrt{k / m}$.

At a distance $d \sqrt{2}$ is the next nearest particle, so at this distance the interaction force should be zero. In total, each particle has a maximum of 12 nearest neighbors, which must be taken into account when calculating the sum of the interaction forces, this accelerates the computation in comparison with a rectangular grid where it is necessary to process 26 neighbors.

\section{Numerical modeling}

Let us consider a numerical model of the propagation of acoustic waves in a solid in which the scattering object (heterogeneity) represents the plate at an angle, and the background medium is the equivalent of brass (sound velocity $4430 \mathrm{~m} / \mathrm{c}$, density $8485 \mathrm{~kg} / \mathrm{m} 3$ ). When carrying out a numerical experiment in the area with dimensions of $128 \mathrm{~mm}$ by $55 \mathrm{~mm}$. In the case of a solid medium, with the propagation of a perturbation in the medium, two types of waves will already be propagated: longitudinal and transverse. This is due to the fact that a shear elasticity exists in a solid medium, in contrast to a liquid one. Figure 3 shows the result of modeling a wave emanating from a particle from above. The red indicates the velocity of the particle along the $\mathrm{X}$ axis, green along the $\mathrm{Y}$ axis, blue along the $\mathrm{Z}$ axis. The initial perturbation was directed along the $\mathrm{X}$ axis. It can be seen that the longitudinal wave is ahead of the transverse wave, the waves are diffracted on heterogeneity, and wave 


\section{IME\&T 2017}

reflection, occurs according to Snellius law (the angle of incidence equal to angle of reflection).

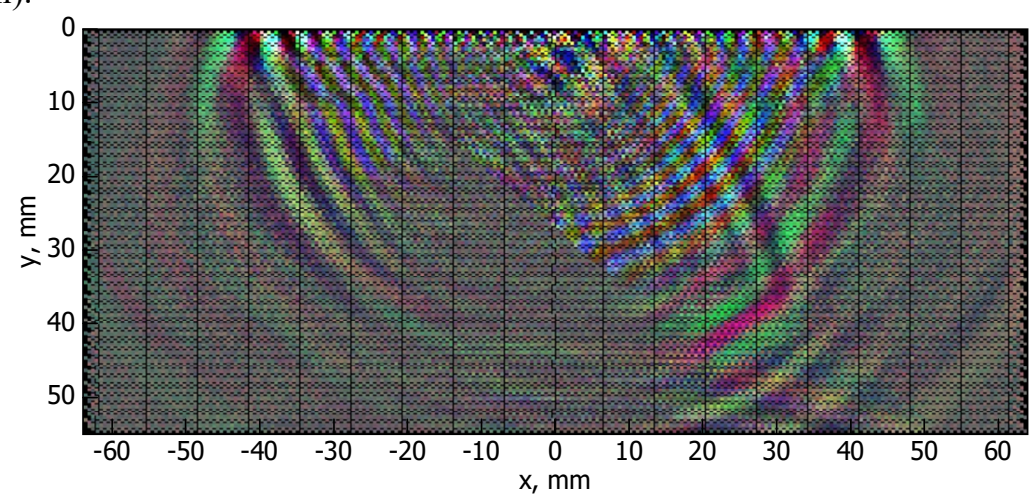

Fig. 3. The result of simulation of oscillations in a lattice after excitation of one particle by a monopolar pulse.

In addition, a numerical simulation of a structure composed of three different solid media was carried out. This structure is an ultrasonic waveguide (Figure 4) made of titanium connected to a piezoelectric element and a reflector made of steel. To specify materials with different properties, the values of the particle mass and the slope of the curve of the dependence of the force on the distance near the equilibrium point were regulated.

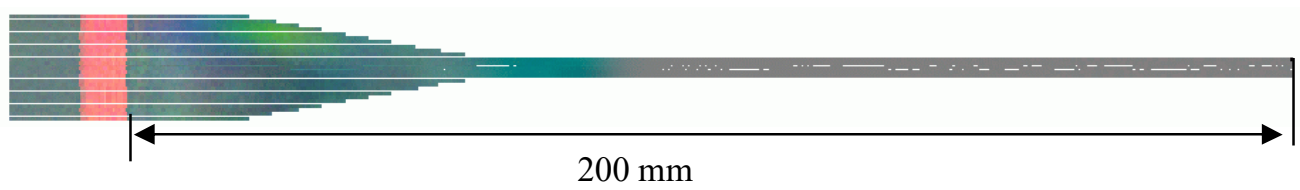

Fig. 4. Model of ultrasonic waveguide.

In this case, the piezoelement is a source of oscillations and its entire volume is subject to external disturbing impact. The model provides additional compressing and stretching of the piezoelement depending on the time, but it interacts with other materials and conducts sound.

As a result of numerical simulation, the values of the resonant frequencies for the given structure were obtained (Figure 5).

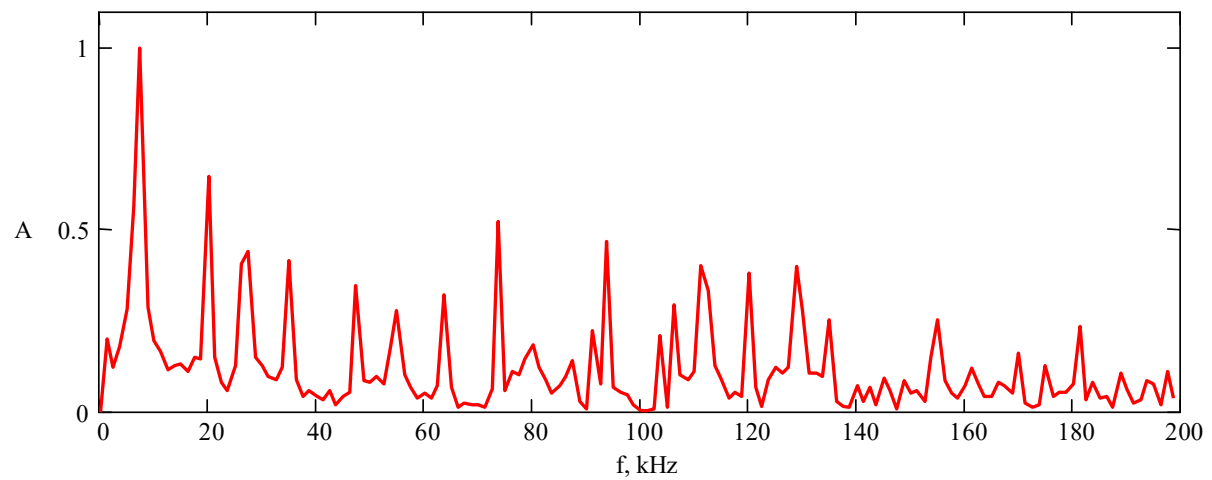

Fig. 5. Spectrum of an impulse signal on the end of a waveguide. 
At a frequency of $20 \mathrm{kHz}$, the amplitude of the oscillations waveguide behaves as shown in Figure 6. It can be seen that when the excitation frequency coincides with the resonant frequency an almost linear increase in the amplitude of the vibrations on the end of the waveguide occurs.

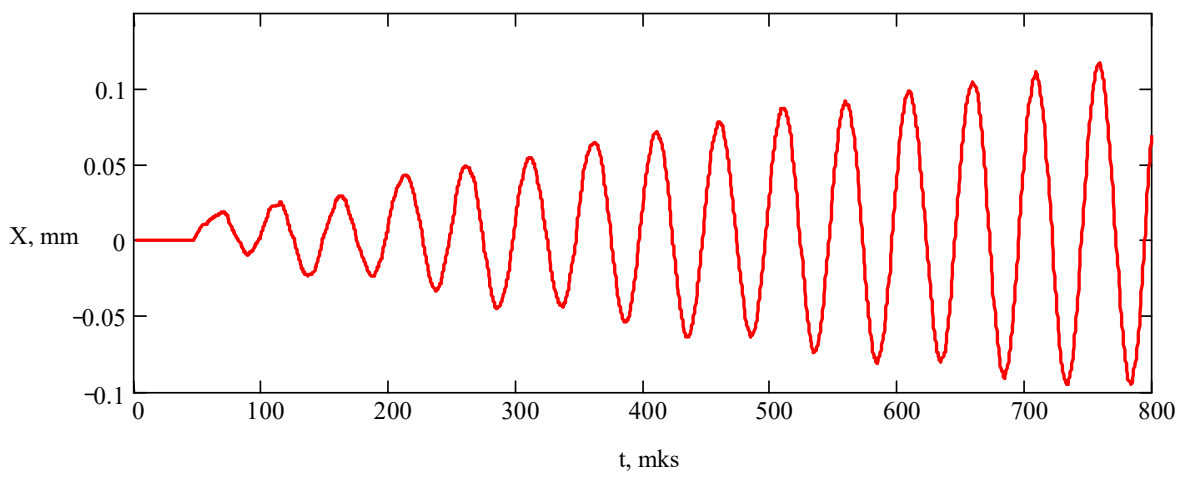

Fig. 6. Harmonic signal at a frequency of $20 \mathrm{kHz}$ on the end of the waveguide.

From the above examples of numerical simulation, one can observe a number of acoustic effects: diffraction, reflection, interference, resonance phenomena in waveguides. In the linear approximation, this model reduces to the wave equation. The peculiarity of the model is that it makes it possible to significantly reduce the calculation time by reducing the number of processed particles. The model allows taking into account nonlinear effects in the medium, by assigning the force dependence graph.

\section{Conclusion}

As a result of the work, a computer model has been created that makes it possible to calculate the process of propagation of an acoustic wave in a solid body in the presence of heterogeneity. Here was shown a possibly of prediction of the diffraction and reflection effects of waves from an object of arbitrary shape.

\section{Acknowledgments}

The work was supported by the Ministry of Education and Science of Russia within the framework of the project "Creation of a high-tech complex of ultrasound surgery" (Unique identifier of the project RFMEF157517X0163).

\section{References}

[1] V.A. Barkhatov, Russian Journal of Nondestructive Testing 43, 605 (2007) doi: $10.1134 / \mathrm{S} 1061830907090069$

[2] R.W. Hockney, J.W.Eastwood, Computer Simulation Using Particles (N. J.: A. Hilger. 1988)

[3] A.M. Krivtsov, N.V. Krivtsova, The Far East. mat. f. 3, 254 (2002)

[4] D.A. Sukhoruchkin, O.A. Sapozhnikov, Proc. of the school-seminar "Waves-2016" 22 (2016) 